\section{Necessidade insatisfeita por métodos anticoncepcionais na Bolívia, 1998}

\author{
Unmet needs for contraceptive \\ methods in Bolivia, 1998
}

1 Centro de Desenvolvimento
e Planejamento Regional,
Universidade Federal
de Minas Gerais,
Belo Horizonte, Brasil.
2 Escola Nacional de Saúde
Pública Sergio Arouca,
Fundação Oswaldo Cruz,
Rio de Janeiro, Brasil.

Correspondência
R. N. Rodrigues
Departamento de
Demografia, Centro
de Desenvolvimento
e Planejamento Regional,
Universidade Federal
de Minas Gerais.
Rua Curitiba 832, 9o andar,
Belo Horizonte, MG
30170-120, Brasil.
beto@cedeplar.ufmg.br

\section{Abstract}

Bolivia, like most developing countries, has experienced a decline in fertility. However, in 1998, as many as $27.6 \%$ of all married women in Bolivia were classified as having an unmet need for contraception, of whom 20.6\% needed to limit and 7\% needed to space an additional child. The current article analyzes the unmet need for contraception in Bolivia. A multinomial hierarchical model was used to identify factors associated with the unmet need for contraception. Variables such as woman's age, number of living children, length of marriage, region, and schooling were strongly associated with unmet need for contraception. Despite the large number of variables in the model, a certain amount of unexplained variation at the individual level still remains, indicating the need to incorporate more detailed analysis at the community level.

Contraception; Birth Control (Public Health); Fertility

\author{
Moisés Calle ${ }^{1}$ \\ Roberto Nascimento Rodrigues 1 \\ Iúri da Costa Leite 2
}

\section{Introdução}

As condições de saúde da Bolívia são típicas de um país situado entre os mais pobres do mundo 1 . A despeito de melhorias dos indicadores de saúde, principalmente devido a um aumento de investimentos no setor, da ordem de $80 \%$, entre 1987 e 1995, esses indicadores ainda se encontram em patamares bastante elevados quando comparados aos de outros países da América Latina. Em 1998, as taxas de mortalidade infantil e mortalidade materna situavamse, respectivamente, em torno de 67 por mil e 371 por 100 mil nascidos.

Durante esse período (1987/1995), a taxa de fecundidade total declinou de 5,6 para 4,2 filhos, possivelmente em função do aumento da prevalência de métodos anticoncepcionais, que passou de $24 \%$ para $45 \%$ entre 1983 e 19942 . A prevalência do uso de métodos anticoncepcionais continuou aumentando na segunda metade dos anos 90, embora em ritmo menos acelerado, tendo atingido, em 1998, o patamar de $48 \%$ entre as mulheres em união 3,4 .

A taxa de fecundidade total, na Bolívia, pode ser considerada elevada, sob vários pontos de vista, sobretudo quando se observa que boa parte dos nascimentos é considerada pelas mulheres como não desejados, seja porque não estavam planejados para o momento em que ocorreram, seja porque elas não queriam mais outro filho adicional. O percentual de mulhe- 
res em união usuárias de métodos anticoncepcionais (48\%) está bem aquém do que seria de esperar, tendo em vista o desejo manifesto pelas mulheres de espaçar ou limitar sua fecundidade 4 . Dessas mulheres, apenas pouco mais da metade $(52,1 \%)$ usava algum tipo de método anticoncepcional moderno 3 . O método mais utilizado era a abstinência $(41,5 \%)$, considerado menos eficaz no controle da fecundidade 5,6,7, seguido de longe pelo DIU (23\%), esterilização feminina $(13,4 \%)$ e pílula $(7,8 \%)$.

É certo que uma parcela dessa discrepância entre o número de filhos desejados e aquele efetivamente tido deve-se, em parte, a falhas decorrentes da ineficácia do método anticoncepcional ou de sua má utilização. Mas também é certo que parte dessa divergência é oriunda de restrições ao acesso a métodos contraceptivos, o que configura uma demanda insatisfeita por programas de atenção à saúde reprodutiva da mulher.

Bongaarts \& Bruce 8 ressaltam que, em vários países em desenvolvimento, uma elevada proporção de mulheres que desejam limitar ou espaçar os nascimentos não combina tal desejo com o uso efetivo de métodos anticoncepcionais. Essas mulheres são classificadas como tendo uma necessidade insatisfeita por contracepção.

O objetivo deste estudo é conhecer a magnitude da necessidade insatisfeita por métodos anticoncepcionais entre as mulheres bolivianas, assim como os seus fatores condicionantes. Essas informações se constituem em elementos essenciais para uma correta formulação e implementação de políticas de atenção à saúde reprodutiva, pautadas na geração de condições adequadas para que os casais possam transformar, em realidade, o seu tamanho ideal de família.

\section{Material e métodos}

\section{Fonte de dados}

A fonte básica de informação utilizada no presente estudo é a Encuesta Nacional de Demografia y Salud, realizada na Bolívia, em 1998 (ENDSA, 1998), pelo Instituto Nacional de Estatística (INE) 3 . Esta pesquisa faz parte da terceira fase do Programa Mundial de Pesquisas de Demografia e Saúde (DHS) e propiciou informações sobre 11.187 mulheres de 15 a 49 anos, das quais, 6.649 estavam casadas ou em união ${ }^{3}$.

\section{Conceito e estimativa}

de necessidade insatisfeita

O conceito de necessidade insatisfeita foi primeiro formulado com base nos dados coletados pelas World Fertility Surveys (WFS), durante os anos 60 e 70, com o objetivo de prover informações sobre conhecimento, atitudes e práticas relacionadas à fecundidade.

A despeito dessa discussão, o estudo da necessidade insatisfeita tem ganhado destaque nas tentativas de abordagem e entendimento da dinâmica reprodutiva em países em desenvolvimento. A idéia é tentar traduzir a motivação das mulheres em relação ao seu comportamento reprodutivo, expresso no desejo de adiar ou evitar um futuro nascimento. Para isso, é importante estabelecer, com clareza, qual é o universo de mulheres consideradas na análise, uma vez que não devem ser incluídas aquelas que, por diferentes razões, não estão expostas ao risco de engravidar.

A dificuldade em avaliar-se a exposição ao risco de engravidar entre as solteiras é a principal barreira para inclusão dessas mulheres na metodologia de cálculo. Nesse sentido, utilizase a metodologia desenvolvida por Westoff 9 , que define como tendo necessidade insatisfeita por anticoncepção o conjunto de mulheres casadas ou em união, férteis, não usuárias de métodos anticoncepcionais, assim como as grávidas ou amenorréicas que não planejaram a sua atual ou última gravidez e não estavam usando método anticoncepcional quando engravidaram. Ressalta-se aqui que, com base em informações fornecidas sobre desejo por mais filhos e o momento de tê-los, classifica-se a necessidade insatisfeita como sendo para limitar (quando a mulher não deseja outro filho adicional) ou espaçar nascimentos (quando a mulher deseja outro filho adicional no futuro).

\section{Metodologia}

Para avaliar os fatores sócio-demográficos associados com a necessidade insatisfeita por anticoncepção, utiliza-se o modelo multinomial, pois a variável resposta é composta de três categorias nominais: (1) necessidade para espaçar, (2) necessidade para limitar, e (3) sem necessidade 10,11. Além disso, um parâmetro aleatório, referente à área onde as mulheres residem, é incluído no modelo com vista a captar a estrutura hierárquica nos dados da ENDSA. Segundo Steele \& Curtis 12, mulheres selecionadas de uma mesma área geográfica são mais semelhantes em suas atitudes em relação ao uso de métodos anticoncepcionais do que mu- 
lheres selecionadas de áreas diferentes. Isso gera uma estrutura hierárquica nos dados que resulta em correlação entre as observações 13,14. Quando a estrutura hierárquica é ignorada, os erros-padrão associados às estimativas dos parâmetros do modelo tendem a ser subestimados e, assim, pode-se selecionar uma variável como estatisticamente significativa quando ela, de fato, não é 7,14 .

O modelo hierárquico multinomial utilizado neste estudo é definido da seguinte forma:

$$
\ln \left(\frac{\pi_{i j s}}{\pi_{i j 3}}\right)=\beta_{k}^{\prime} x_{i j}+u_{j}, s=1,2,
$$

no qual: $\pi_{i j s}$ é a probabilidade de uma mulher i residente na localidade $\mathrm{j}$ ser classificada na categoria $\mathrm{s}$ (necessidade para espaçar, $\mathrm{s}=1$; e necessidade para limitar, $\mathrm{s}=2$ ) e $\pi_{i j 3}$ é a probabilidade de ser classificada na categoria de referência 3, que é composta por mulheres sem necessidade por anticoncepção. O termo aleatório $u_{j}$ é incluído para captar o efeito da correlação entre as observações de uma mesma localidade.

Cabe ressaltar que o modelo hierárquico foi utilizado apenas para controlar a correlação entre as observações. Nada foi explorado além disso, visto que não é possível identificar, com base nas informações disponíveis, as áreas onde as mulheres residem. O programa MIXNO, de domínio público (http://tigger.uic.edu/ hedeker/mix.html), foi utilizado para estimar os parâmetros do modelo.

Um conjunto de variáveis sócio-demográficas foi selecionado, privilegiando aquelas que têm sido apontadas como associadas com a necessidade insatisfeita por anticoncepção 15,16, 17,18: idade, número de filhos, nível educacional, situação de residência (rural, urbano), duração do casamento, condição de atividade (trabalha ou não), exposição a meios de comunicação (assiste à TV), medidas de relações de gênero (diálogo sobre planejamento familiar) e tipo de família (nuclear, composta e estendida).

\section{Estimativa da necessidade insatisfeita por anticoncepção}

A seleção do universo amostral, que envolve a discussão conceitual acerca da necessidade insatisfeita e da exposição ao risco de engravidar, pode ser acompanhada pela ilustração contida na Figura 1. No início, foi selecionado o conjunto formado pelas mulheres em união, em idade reprodutiva (15-49 anos). Em seguida, foram eliminadas, progressivamente, as categorias de mulheres que não estariam com ne- cessidade insatisfeita de uso de métodos contraceptivos. Primeiro, foram excluídas as mulheres usuárias de métodos anticoncepcionais $(48,3 \%)$, seja para espaçar $(13,3 \%)$ ou para limi$\operatorname{tar}(35 \%)$ o número de filhos, independentemente de estarem usando método moderno ou tradicional. Entre as mulheres não usuárias $(51,7 \%)$ e, portanto, potencialmente passíveis de serem classificadas como tendo necessidade insatisfeita por anticoncepção, distinguem-se dois grupos: grávidas ou amenorréicas $(22,2 \%)$ e não grávidas ou amenorréicas $(29,5 \%)$.

O primeiro grupo, composto por grávidas ou amenorréicas, foi subdividido com base na declaração das mulheres por ocasião da entrevista. Aquelas cuja última gravidez ocorreu antes do período previsto foram classificadas como em necessidade de método anticoncepcional para espaçar $(4,7 \%)$, ao passo que as mulheres cuja última gravidez não era desejada em nenhum período, porque elas não queriam mais ter filhos, foram classificadas como em necessidade de método contraceptivo para limitar (8,2\%). Já as mulheres que declararam que desejavam sua última gravidez no período em que ficaram grávidas foram classificadas como sem necessidade de uso de método contraceptivo $(9,4 \%)$

O segundo grupo (não grávidas ou amenorréicas) pode ser subdivido em dois subgrupos: fecundas e infecundas. O critério adotado para definir a condição de fertilidade das mulheres se ajusta à disponibilidade de informações contidas na DHS e aos critérios estabelecidos por Westoff 9 . As mulheres classificadas como infecundas $(10,5 \%)$ foram aquelas que não tiveram filhos nos últimos cinco anos, embora tenham estado em união contínua sem ter usado métodos anticoncepcionais durante esse período. Também foram consideradas inférteis aquelas mulheres cuja menstruação não retornou nos últimos seis meses anteriores à data da pesquisa, assim como aquelas que declararam estar em menopausa ou, quando indagadas sobre quando iniciou a sua última menstruação, declararam que jamais tinham menstruado. Algumas mulheres, cerca de $1,1 \%$, não puderam ser classificadas como fecundas ou infecundas. Assumindo-se, então, que todas elas são infecundas, uma vez que seguindo outro critério, menos rígido, definido pela DHS, $80 \%$ estariam nessa condição, pode-se dizer que, em 1998, a proporção de mulheres em união, na Bolívia, que podem ser consideradas como infecundas deve variar entre $10,5 \%$ e $11,6 \%(10,5 \%+1,1 \%)$. No subgrupo das mulheres fecundas $(17,8 \%)$, cerca de $3,1 \%$ declararam que desejavam um filho adicional já, o que significa dizer que elas 


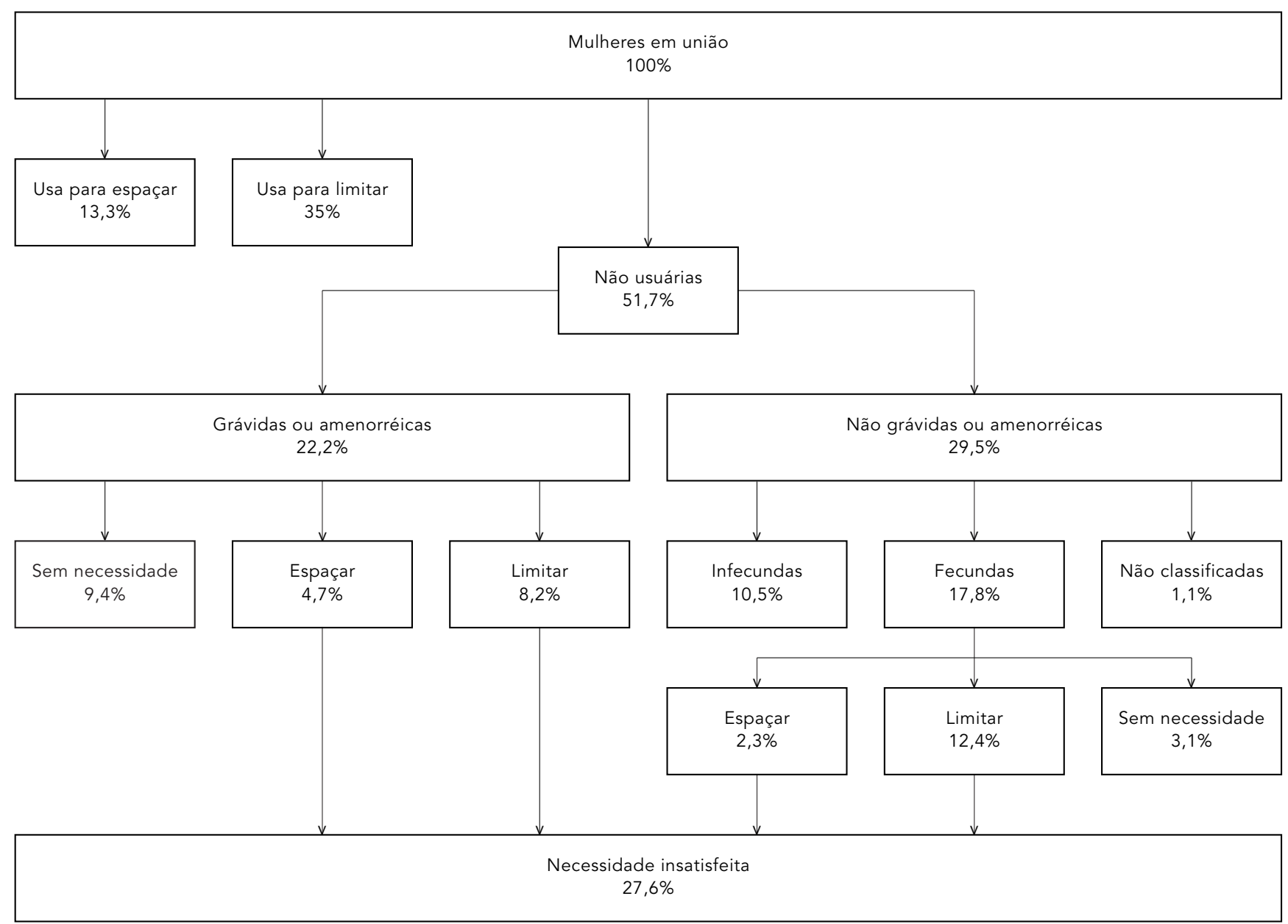

foram classificadas como sem necessidade de utilização de métodos anticoncepcionais. As demais desejavam espaçar $(2,3 \%)$ ou limitar $(12,4 \%)$ o número de filhos. Em suma, observou-se que $27,6 \%$ das mulheres bolivianas, em união, entre 15 e 49 anos, tinham necessidade insatisfeita por métodos anticoncepcionais (8\% para espaçar e 19,6\% para limitar). Trata-se de um nível consideravelmente elevado. Em 1991, no Nordeste do Brasil, quando a taxa de fecundidade era de 3,8 filhos por mulher, a proporção de necessidade insatisfeita era de $17,6 \% 16$.

\section{Resultados}

A Tabela 1 apresenta os coeficientes do modelo parcimonioso de necessidade insatisfeita por anticoncepção. Três variáveis foram excluídas do modelo, pois não foram estatisticamente significativas ao nível de 5\%: situação de residência, condição de atividade e tipo de família. A variável idade foi incluída como uma função quadrática, mas apenas o termo linear teve efeito sobre a chance de ter uma necessidade insatisfeita. No caso da variável duração do casamento, o termo quadrático mostrou-se estatisticamente significativo. As demais variáveis foram incluídas como categóricas. Para facilitar a interpretação, foram calculadas probabilidades acumuladas para cada variável, mantendo as demais constantes no seu valor médio. Essas probabilidades estão apresentadas na Tabela 2 .

A idade da mulher apresenta uma forte associação com a necessidade insatisfeita. Aumentos sucessivos de idade resultam em uma tendência crescente de necessidade para limi- 
tar e decrescente de necessidade para espaçar. $\mathrm{Na}$ medida em que as mulheres envelhecem eleva-se a probabilidade de que elas tenham atingido o tamanho ideal de família e, portanto, de demandarem o uso de métodos anticoncepcionais para limitar a fecundidade. Já a probabilidade de não necessitar de planejamento familiar apresenta valores bastante similares para todos os grupos etários.

O padrão registrado para duração de casamento é positivo e crescente no caso da necessidade para limitar, ligeiramente côncavo quando se considera a necessidade para espaçar e, convexo em relação à ausência de necessidade insatisfeita. Até a faixa de duração de casamento de 10-14 anos, é crescente a probabilidade de que as mulheres não necessitem do uso de métodos contraceptivos, e para casamentos que duram além desse tempo, há sucessivas reduções nas probabilidades, possivelmente porque, nessas categorias, há uma proporção elevada de mulheres já esterilizadas, depois de terem completado ou ultrapassado o tamanho ideal de família.

Com relação ao número de filhos vivos, observa-se um padrão diverso entre as probabilidades de necessidade para espaçar e limitar: variação negativa com aumento no número de filhos no primeiro caso e positiva no segundo. Assim, mulheres com quatro filhos ou mais apresentam a mais alta probabilidade de necessitar de métodos anticoncepcionais para limitar e, a mais baixa probabilidade de necessitar de métodos para espaçar. Aqui se configura a mesma lógica interpretativa aventada para a análise de variações decorrentes da idade.

A probabilidade de necessidade insatisfeita para limitar é praticamente igual entre mulheres analfabetas e aquelas com nível de instrução primária, mas se reduz sensivelmente entre mulheres que freqüentaram os cursos secundário e superior. É possível que mulheres de maior nível de instrução tenham maior acesso a métodos eficazes de controle da fecundidade, o que reduz a probabilidade de apresentarem necessidade insatisfeita. Situação oposta deve preponderar no caso de mulheres com menor nível de instrução.

As diferenças nas probabilidades de necessidade insatisfeita segundo exposição a mensagens televisivas e o diálogo com o marido sobre planejamento familiar não apresentam grande variação quando se observa o padrão entre as categorias das variáveis, mas, em todas elas, a probabilidade de necessidade de métodos para limitar é quase sempre mais de duas vezes maior do que a necessidade de métodos para espaçar.
Tabela 1

Coeficientes estimados pelo modelo hierárquico multinomial para a necessidade insatisfeita por anticoncepção segundo características selecionadas, Bolívia, 1998.

\begin{tabular}{|c|c|c|}
\hline \multirow[t]{2}{*}{ Características } & \multicolumn{2}{|c|}{ Necessidade insatisfeita } \\
\hline & Espaçar & Limitar \\
\hline Idade da mulher & $-0,051$ & 0,022 \\
\hline \multicolumn{3}{|c|}{ Número de filhos vivos } \\
\hline 0 & 0,000 & 0,000 \\
\hline 1 & $1,064^{*}$ & $1,430^{*}$ \\
\hline 2 & $1,427^{\star}$ & $2,692^{*}$ \\
\hline 3 & $1,588^{*}$ & $3,198^{*}$ \\
\hline 4 ou + & $1,931^{*}$ & $4,140^{*}$ \\
\hline \multicolumn{3}{|c|}{ Duração do casamento } \\
\hline Duração & $-0,104^{*}$ & $-0,049$ \\
\hline Duração2 & $0,004^{\star}$ & $0,003^{*}$ \\
\hline \multicolumn{3}{|l|}{ Nível de instrução } \\
\hline Nenhuma & 0,000 & 0,000 \\
\hline Primária & 0,185 & 0,104 \\
\hline Secundária & 0,200 & $-0,312$ \\
\hline Superior & $-0,039$ & $-1,181 *$ \\
\hline \multicolumn{3}{|l|}{ Região } \\
\hline Vales & 0,000 & 0,000 \\
\hline Plano Alto & $-0,348^{*}$ & $-0,469$ \\
\hline Lhanos & $-0,219$ & $-1,155^{\star}$ \\
\hline \multicolumn{3}{|l|}{ Assiste à TV } \\
\hline Não & 0,000 & 0,000 \\
\hline Sim & $0,317^{\star}$ & 0,236 \\
\hline \multicolumn{3}{|c|}{$\begin{array}{l}\text { Conversa com o marido } \\
\text { sobre planejamento familiar }\end{array}$} \\
\hline Não & 0,000 & 0,000 \\
\hline $1-2$ vezes & $-0,101$ & $-0,038$ \\
\hline 3 ou + vezes & $-0,382$ & $-0,513^{*}$ \\
\hline Efeito aleatório & $0,292^{*}$ & 0,794 * \\
\hline
\end{tabular}

Duração2 = a variável duração do casamento foi incluída como função quadrática. * Significância estatística $\mathrm{p}<0,05$.

Fonte: Encuesta Nacional de Demografía y Salud 3.

Os efeitos aleatórios foram estatisticamente significativos nas duas equações, reforçando a necessidade de incorporar-se a estrutura hierárquica no modelo.

\section{Discussão}

Gradativamente, o planejamento familiar tem sido um elemento cada vez mais presente nas políticas e programas de população e constitui parte integral da saúde reprodutiva. Em 1998, em nível mundial, 60,9\% das mulheres casadas ou em união utilizavam algum método anticoncepcional, proporção que se eleva para $70,5 \%$ 
Tabela 2

Probabilidades da necessidade de métodos anticoncepcionais, segundo características das mulheres em união. Bolívia, 1998.

\begin{tabular}{|c|c|c|c|}
\hline Característica & $\begin{array}{l}\text { Necessita } \\
\text { espaçar }\end{array}$ & $\begin{array}{l}\text { Necessita } \\
\text { limitar }\end{array}$ & Não necessita \\
\hline \multicolumn{4}{|l|}{ Idade (anos) } \\
\hline 15 & 0,355 & 0,369 & 0,276 \\
\hline 20 & 0,285 & 0,428 & 0,287 \\
\hline 25 & 0,224 & 0,485 & 0,291 \\
\hline 30 & 0,173 & 0,538 & 0,289 \\
\hline 35 & 0,131 & 0,587 & 0,282 \\
\hline 40 & 0,098 & 0,630 & 0,272 \\
\hline 45 & 0,072 & 0,669 & 0,259 \\
\hline \multicolumn{4}{|c|}{ Número de filhos vivos } \\
\hline 0 & 0,104 & 0,078 & 0,818 \\
\hline 1 & 0,209 & 0,224 & 0,567 \\
\hline 2 & 0,181 & 0,478 & 0,341 \\
\hline 3 & 0,165 & 0,571 & 0,264 \\
\hline $4 \mathrm{ou}+$ & 0,112 & 0,760 & 0,128 \\
\hline \multicolumn{4}{|c|}{ Duração do casamento (anos) } \\
\hline 0 & 0,231 & 0,503 & 0,266 \\
\hline 5 & 0,180 & 0,504 & 0,316 \\
\hline 10 & 0,151 & 0,517 & 0,331 \\
\hline 15 & 0,139 & 0,551 & 0,310 \\
\hline 20 & 0,138 & 0,605 & 0,257 \\
\hline 25 & 0,145 & 0,670 & 0,185 \\
\hline 30 & 0,158 & 0,729 & 0,113 \\
\hline \multicolumn{4}{|c|}{ Nível de instrução } \\
\hline Nenhuma & 0,140 & 0,582 & 0,279 \\
\hline Primária & 0,154 & 0,591 & 0,255 \\
\hline Secundária & 0,195 & 0,487 & 0,318 \\
\hline Superior & 0,227 & 0,302 & 0,471 \\
\hline \multicolumn{4}{|l|}{ Região } \\
\hline Vales & 0,149 & 0,639 & 0,212 \\
\hline Plano Alto & 0,150 & 0,555 & 0,295 \\
\hline Lhanos & 0,222 & 0,379 & 0,399 \\
\hline \multicolumn{4}{|c|}{$\begin{array}{l}\text { Conversa com o marido sobre } \\
\text { planejamento familiar }\end{array}$} \\
\hline Não & 0,173 & 0,556 & 0,271 \\
\hline $1-2$ vezes & 0,163 & 0,556 & 0,281 \\
\hline 3 ou + vezes & 0,164 & 0,461 & 0,375 \\
\hline
\end{tabular}

* Significância estatística $p<0,05$.

Fonte: Encuesta Nacional de Demografía y Salud 3.

no caso de mulheres residentes na América Latina e no Caribe. Quando são considerados apenas os métodos modernos, essas proporções chegam a $54 \%$ em todo o mundo e, a $61,7 \%$ na América Latina e no Caribe 19.

A maioria dos usuários de métodos anticoncepcionais opta por métodos modernos, que correspondem a $90 \%$ do uso de contraceptivos em todo o mundo. Em particular, três métodos anticoncepcionais femininos são os mais utilizados: esterilização feminina, DIU e pílulas. Em relação ao uso de métodos específicos, existem diferenças entre as regiões mais e menos desenvolvidas. Por exemplo, os usuários de anticoncepcionais nas regiões mais desenvolvidas preferem métodos de efeito curto e reversível, ao passo que os casais nas regiões menos desenvolvidas se inclinam por métodos de efeito prolongado e de grande eficácia clínica. Aproximadamente, $1 / 3$ dos países recorre, em geral, unicamente a um método, a esterilização ou a pílula, que corresponde à metade do uso de contraceptivos 19.

Na Bolívia, em 1998, a despeito do reduzido percentual de mulheres esterilizadas, em comparação com os níveis registrados em outros países em desenvolvimento, uma proporção considerável de mulheres em união (35\%) estava usando métodos anticoncepcionais para limitar o número de filhos, ao passo que, em apenas $13,3 \%$ dos casos, a utilização de métodos contraceptivos tinha o objetivo de espaçar os nascimentos. De qualquer forma, trata-se de um panorama coerente com o nível elevado de fecundidade da Bolívia. Esse certamente é um ponto de investigação importante para elucidar a dinâmica reprodutiva das mulheres bolivianas. Nesse sentido, seria interessante saber, por exemplo, qual o percentual de gravidez indesejada decorrente de falha no uso de métodos, especialmente levando-se em consideração que o método mais utilizado pelas mulheres bolivianas continua sendo a abstinência.

A análise empreendida ao longo deste artigo revelou que, a despeito do processo de redução dos níveis de fecundidade na Bolívia, a proporção de mulheres com necessidade insatisfeita de planejamento familiar é consideravelmente elevada, especialmente quando se considera a necessidade para limitar o tamanho da família. Quase $28 \%$ das mulheres em união apresentaram necessidade insatisfeita de métodos contraceptivos, para espaçar (7\%) ou para limitar $(21 \%)$ o nascimento de filhos adicionais. Esse resultado, combinado com a constatação de que o método contraceptivo mais utilizado pelas mulheres bolivianas é a abstinência, pouco eficaz no controle da fecundidade, ressalta a importância do entendimento da dinâmica reprodutiva da Bolívia para subsidiar o desenho e a implementação de políticas e ações visando oferecer à população meios mais eficazes para planejar satisfatoriamente o tamanho de família.

A idade da mulher e o número de filhos apresentam uma relação direta com a necessidade 
insatisfeita para limitar e uma relação inversa para espaçar. O padrão descrito pela variável duração do casamento é positivo e crescente no caso da necessidade para limitar, ligeiramente côncavo quando se considera a necessidade para espaçar e, convexo em relação à ausência de necessidade insatisfeita.

A proporção de mulheres com necessidade insatisfeita para limitar é praticamente igual entre as mulheres analfabetas e as com nível de instrução primária, mas se reduz sensivelmente entre as mulheres com cursos secundário e superior.

\section{Considerações finais}

Apesar do grande número de fatores incluídos no modelo, observa-se que ainda permanece um significativo volume de variação não explicada ao nível individual, apontando para a necessidade de um detalhamento maior de análises ao nível da comunidade, incluindo um conjunto de variáveis explicativas, como a presen-

Resumo

A Bolívia, como grande parte dos países em desenvolvimento, tem experimentado um processo de redução da fecundidade. No entanto, em 1998, estima-se que 27,6\% das mulheres bolivianas em união tinham necessidade insatisfeita por métodos anticoncepcionais; dentre as quais, 20,6\% tinham necessidade para limitar, e 7\%, para espaçar o nascimento de um filho adicional. Neste artigo, analisa-se a necessidade insatisfeita por métodos anticoncepcionais na Bolívia. Um modelo multinomial hierárquico foi utilizado para identificar fatores associados com a necessidade insatisfeita de métodos anticoncepcionais. Variáveis como idade, número de filhos, duração de casamento, região e nível de instrução apresentaram forte associação com a necessidade insatisfeita por anticoncepção na Bolívia. Apesar do grande número de fatores incluídos no modelo, ainda permanece um expressivo volume de variação não explicada ao nível individual, apontando para a necessidade de um maior detalhamento de análises ao nível da comunidade.

Anticoncepção; Controle de Natalidade (Saúde Pública); Fertilidade ça de programas de planejamento familiar ou a exposição a serviços de saúde. Por outro lado, na maioria dos países em desenvolvimento que dispõem de dados sobre tendências, observase que, nos últimos dez anos, tem havido um aumento substancial do uso de anticoncepcionais, provavelmente devido, em parte, às campanhas de promoção do uso de preservativo para proteção contra a contaminação pelo HIV.

Ainda assim, há sérias críticas à falta de sensibilidade dos profissionais de saúde em relação às diferenças culturais, afastando, assim, uma parcela significativa das mulheres, principalmente as mais pobres, de baixa escolaridade, dos hospitais e clínicas 5 . A formulação de políticas públicas adequadas para possibilitar o acesso e a escolha de métodos adequados de planejamento familiar requer uma análise crítica da oferta de serviços de saúde e de planejamento familiar, com ênfase especial na qualidade dos serviços e no respeito aos direitos reprodutivos de homens e mulheres, de acordo com os princípios estabelecidos na Conferência do Cairo 20.

\section{Colaboradores}

M. Calle, R. N. Rodrigues e I. C. Leite participaram da revisão de literatura, elaboração da metodologia, análise dos resultados e redação do artigo final. 


\section{Referências}

1. Olave M. Financing reproductive health in Bolivia. Washington DC: Policy Project; 2000.

2. Hubner VC, Murillo AG, Ballivián MP, André R, Barbery XM, Castro MD, et al. Diagnóstico cualitativo de la atención en salud reproductiva en Bolivia. Genebra: Organización Mundial de la Salud; 1996.

3. Sardán MG, Ochoa LH, Vargas AG, editores. Encuesta nacional de demografía y salud. La Paz: Instituto Nacional de Estadística; 1998.

4. Calle M. Estudo dos componentes da dinâmica reprodutiva na Bolívia, 1998 [Tese de Doutorado]. Belo Horizonte: Centro de Desenvolvimento e Planejamento Regional, Universidade Federal de Minas Gerais; 2003.

5. Shuler R, Choque ME, Rance S. Misinformation, mistrust, and mistreatment: family planning among Bolivian market women. Stud Fam Plann 1994; 25:1-19.

6. Blanc AK, Curtis SL, Croft TN. Monitoring contraceptive continuation: links to fertility outcomes and quality care. Stud Fam Plann 2002; 33:127-40.

7. Leite IC. Descontinuação de métodos anticoncepcionais no Nordeste do Brasil, 1986-1991. Cad Saúde Pública 2003; 19:1005-16.

8. Bongaarts J, Bruce J. The causes of unmet need for contraception and the social content of services. Stud Fam Plann 1995; 26:57-75.

9. Westoff $C$. The potential demand for family planning: a new measure of unmet need and estimates for five Latin American countries. Int Fam Plan Perspect 1988; 14:45-53.

10. Agresti A. Categorical data analysis. New York: John Wiley \& Sons; 1990.

11. Retherford RD, Choe MK. Statistical models for causal analysis. New York: John Wiley \& Sons; 1993.

12. Steele F, Curtis SL. Appropriate methods for analyzing the effect of method choice on contraceptive discontinuation. Demography 2003; 40:1-22.
13. Bryk AS, Raudenbush SW. Hierarchical linear models. Newbury Park: Sage Publications; 1992.

14. Goldstein H. Multilevel statistical models. London: Edward Arnold; 1995.

15. Westoff CF, Bankole A. Unmet need: 1990-1994. Maryland: Macro International Inc.; 1995. (DHS Comparative Studies, 16).

16. Leite IC. Contraceptive dynamics in Northeast Brazil [Ph.D. Thesis]. Southampton: Department of Social Statistics, University of Southampton; 1998.

17. Nazar-Beutelspacher A, Molina-Rosales D, Salvatierra-Izaba B, Apata-Martelo E, Halperin D. Education and nonuse of contraceptives among poor women in Chiapas, Mexico. Int Fam Plan Perspect 1999; 25:132-8.

18. Wolff B, Blanc AK, Ssekamatte-Ssebuliba J. The role of couple negotiation in unmet need for contraception and decision to stop childbearing in Uganda. Stud Fam Plann 2000; 3:124-37.

19. United Nations. Review and appraisal of the progress made in achieving the goals and objectives of the Programme of Action of the International Conference on Population and Development: the 2004 report. New York: United Nations; 2004.

20. Programa das Nações Unidas para o Desenvolvimento. Direitos reprodutivos e saúde reprodutiva. In: Programa das Nações Unidas para o Desenvolvimento, organizador. Relatório da Conferência Internacional sobre População e Desenvolvimento. Brasília: Fundo de População das Nações Unidas; 1994. p. 57-71.

Recebido em 09/Dez/2005

Versão final reapresentada em 17/Fev/2006 Aprovado em 10/Mar/2006 